\title{
Prevalence of non-syndromic orofacial clefts: based on $15,094,978$ Chinese perinatal infants
}

\author{
Dazhi Fan ${ }^{1,2}$, Shuzhen Wu ${ }^{1,2}$, Li Liu ${ }^{3,5}$, Qing Xia ${ }^{3,6}$, Guo Tian ${ }^{5}$, Wen Wang ${ }^{2}$, Shaoxin \\ $\mathrm{Ye}^{1,2}$, Lijuan Wang ${ }^{2}$, Jiaming Rao ${ }^{1,7}$, Xiao Yang ${ }^{3,8}$, Zhen Yu ${ }^{9}$, Lihong Xin ${ }^{10}$, Song $\mathbf{L i}^{11}$, \\ Zhenghua Duan ${ }^{12}$, Tianchen $\mathrm{Zhang}^{13}$, Song $\mathrm{Wu}^{4}$, Xiaoling $\mathrm{GuO}^{1,2}$ and Zhengping \\ Liu ${ }^{1,2}$ \\ ${ }^{1}$ Foshan Institute of Fetal Medicine, Southern Medical University Affiliated Maternal and Child Health Hospital of Foshan, \\ Foshan, Guangdong, 528000, China \\ ${ }^{2}$ Department of Obstetrics, Southern Medical University Affiliated Maternal and Child Health Hospital of Foshan, Foshan, \\ Guangdong, 528000, China \\ ${ }^{3}$ Department of Epidemiology and Biostatistics, School of Public Health, Anhui Medical University, Hefei, Anhui, 230032, \\ China \\ ${ }^{4}$ School of Integrated Traditional and Western Medicine, Anhui University of Chinese Medicine, Hefei, Anhui, 230038, China \\ ${ }^{5}$ Department of Library, The First Affiliated Hospital, College of Medicine, Zhejiang University, Hangzhou, Zhejiang, 310003, \\ China \\ ${ }^{6}$ Menzies Institute for Medical Research, University of Tasmania, Private Bag 23, Hobart, Tasmania 7000, Australia \\ ${ }^{7}$ Department of Epidemiology, Medical College of Jinan University, Guangzhou, Guangdong, 510632, China \\ ${ }^{8}$ Changzhou Center for Disease Control and Prevention, Changzhou, Jiangsu, 213003, China \\ ${ }^{9}$ Department of Maternal, Child and Adolescent Health, School of Public Health, Anhui Medical University, Hefei, Anhui, \\ 230032, China \\ ${ }^{10}$ Department of Neurology, The Second Affiliated Hospital of Soochow University, Suzhou, Jiangsu 215004, China \\ ${ }^{11}$ Chaohu Hospital Affiliated Anhui Medical University, Chaohu, Anhui, 238000, China \\ ${ }^{12}$ Chengdu Center for Disease Control and Prevention, Chengdu, Sichuan, 610041, China \\ ${ }^{13}$ Department of Communicable Diseases Control, Jiangxi Provincial Center for Disease Control and Prevention, Nanchang \\ 330000, Jiangxi, China
}

Correspondence to: Zhengping Liu, email: liuzphlk81@outlook.com

Xiaoling Guo, email: fsguoxl@163.com

Keywords: non-syndromic orofacial clefts; perinatal infants; prevalence; meta-analysis; China

Received: June 27, $2017 \quad$ Accepted: December 05, $2017 \quad$ Published: January 13, 2018

Copyright: Fan et al. This is an open-access article distributed under the terms of the Creative Commons Attribution License 3.0 (CC BY 3.0), which permits unrestricted use, distribution, and reproduction in any medium, provided the original author and source are credited.

\section{ABSTRACT}

Non-syndromic orofacial clefts (NSOFC), which include cleft lip and palate (CLP), cleft lip only (CLO), and cleft palate only (CPO), contains a range of disorders affecting the lips and oral cavity. No systematic review and meta-analysis has been carried out to synthesize the prevalence of NSOFC in Chinese perinatal infants. We aimed to quantify and understand the variation of prevalence national and regional levels. Four English databases and four Chinese databases were searched using a comprehensive search strategy from inception to April 2017. The random effect model was used for this meta-analysis. To determine the sources of heterogeneity, subgroup analyses and meta-regression were conducted based on different categories. The protocol has been pre-registered in the PROSPERO, number CRD42017062293. 110 studies, including 15,094,978 Chinese perinatal infants, were eligible for inclusion. The pooled prevalence rate for NSOFC was $1.67 \%$ (95\% CI 1.53-1.82), varying with provinces. The pooled prevalence estimate was $0.56 \%(0.50-0.63)$ for CLO, $0.82 \%$ o (0.73-0.90) for CLP, and $0.27 \%$ (0.24-0.30) for CPO. Significant associations were found between overall prevalence estimates and survey year and study region. The 


\section{prevalence of NSOFC was severe in Chinese perinatal infants, varying with provinces. The results will serve as a baseline for future assessment of the overall effectiveness of NSOFC control, and will also support and inform health policy for planning and helping health debates.}

\section{INTRODUCTION}

Non-syndromic orofacial clefts (NSOFC), which include cleft lip and palate (CLP), cleft lip only (CLO), and cleft palate only (CPO), contains a range of disorders affecting the lips and oral cavity $[1,2]$. The etiology and pathogenesis of NSOFC remain largely unknown and involve both genetic and environmental factors contributing to the phenotype [3-5]. It has been shown that these congenital disorders have a significant negative impact on audition, speech, appearance and psychology, which could affect the individuals and their families [6]. Theses impacts can lead to considerable adverse health outcomes and enormous socioeconomic burden [7]. The global average prevalence of NSOFC was approximately $1.7 \%$ in live birth babies [1]. However, the prevalence of NSOFC varies broadly with the difference of ethnicities and geographical positions, and populations of African ancestry having the lowest rates while groups of Amerindian and Asian ancestry possessing the largest [5]. One previous systematic review and meta-analysis emerged in the scientific literature found that the birth prevalence of orofacial clefts was 1.38 per 1000 birth in low- and middle-income countries [8].

China is the biggest developing country and has the largest population in the world. Since mid-1980s, congenital anomalies surveillance systems have been progressively established in hospitals in mainland China [9]. After 30-year effort, all the infants (including live births, stillbirths, abortion or dead fetus) during perinatal period (between 28 gestation weeks and 7 days after birth) will be registered in hospitals. Individual studies have demonstrated that NSOFC (including CLO, CLP, and CPO) is the most common congenital disorders in Chinese perinatal infants [10-12]. Nevertheless, estimates of the prevalence of NSOFC among perinatal infants vary across studies from $0.32 \%$ to $4.70 \%$ o $[13,14]$.

Reliable estimate of prevalence is important for informing efforts to prevent, treat, and identify causes of this disorder among perinatal infants. Hence, the relevant studies are warranted. Meanwhile, many factors, such as survey year, hospital level, sample size, and geographical locations could easily influence the results. We, therefore, aimed to address this research gap by means of a systematic review and meta-analysis of published studies. To the best of our knowledge, this is the first study to determine the prevalence and the characteristics of NSOFC (including CLO, CLP, and CPO) in Chinese perinatal infants. The results will be essential for policymakers and health professionals aware of this influential congenital disorder in Chinese perinatal infants and will be useful for the planning of health services.

\section{RESULTS}

\section{Characteristics of study}

In total, 11967 studies were identified using the initial literature search. The search results were as follows: PubMed $(n=390)$, Elsevier Science Direct $(n=6868)$, Web of Science $(n=578)$, Cochrane Library $(n=40)$, CBM $(n=764)$, CNKI $(n=1133)$, Chongqing VIP $(n$ $=895)$, and WanFang $(n=1299)$. After removing 4032 duplicates and 7383 apparently irrelevant citations by title and abstract review, 552 potentially eligible articles and were reviewed in full-text level. After carefully reading, 442 of them were excluded for various reasons (including 7 duplicates, 25 review articles, 29 diagnostic criteria different, 41 not perinatal infants, 47 community-based survey and 293 accurate data not reported). Finally, a total of 110 articles, including 138 survey researches, met the inclusion criteria in the final meta-analysis (Figure 1).

Detailed characteristics of each included study were shown in Supplementary Table 1. Twenty-nine studies with regarding to the prevalence of NSOFC in Chinese perinatal infants were implemented in East China (4 in Anhui, 2 in Fujian, 5 in Jiangsu, 3 in Jiangxi, 6 in Shandong, 3 in Shanghai, 6 in Zhejiang), 12 in North China (2 in Beijing, 2 in Hebei, 2 in Inner Mongolia, 3 in Shanxi, 3 in Tianjin), 16 in Northwest China (2 in Gansu, 6 in Ningxia, 2 in Qinghai, 3 in Shaanxi, 3 in Xinjiang), 16 in South China (11 in Guangdong, 4 in Guangxi, 1 in Hainan), 13 in Central China (3 in Henan, 3 in Hubei, 7 in Hunan), 10 in Northeast China (2 in Heilongjiang, 4 in Jilin, 4 in Liaoning), and 10 in Southwest China (1 in Chongqing, 2 in Guizhou, 2 in Sichuan, 1 in Tibet, 4 in Yunnan).

A total of 110 articles including 15,094,978 Chinese perinatal infants with a mean number of 137,227 were included. The sample sizes of the included articles ranged from 4192 to 1395155 . The articles were published from 1989 to 2017. Of the 110 eligible articles, the mean NOS score was 7.83, with 24 studies scored 9, 45 studies scored 8, 39 studies scored 7, and 2 studies scored 6 .

\section{Meta-analysis}

Seventy-eight articles, which were constituted of 106 surveys, have reported the prevalence of NSOFC based on the data of 9476601 Chinese perinatal infants. The prevalence of NSOFC varied from $0.32 \%$ o $95 \%$ confidence interval [CI], 0.28-0.35) [13] to $4.70 \%$ (95\% CI, 3.45-5.95) [14]. The meta-analysis revealed significantly high heterogeneity across studies $\left(I^{2}=\right.$ 
$97.3 \%, p<0.001)$. Using a random effects model, the pooled point prevalence of NSOFC was $1.67 \%$ o (95\% CI, $\left.1.53-1.82 ; I^{2}=97.3 \%\right)$. Sensitivity analysis showed that the pooled prevalence was between $1.66 \%$ o (95\% CI, $1.51-$ 1.80) (after excluding Zhang JX et al. [14]) and 1.69\%o (95\% CI, $1.54-1.83$ ) (after excluding Yi YT et al. [15]). These similar results indicated that no individual study affected the overall pooled estimate of the meta-analysis. As shown in Supplemenatry Figure 1, although the funnel plot was slightly asymmetrical, no publication bias was found according to the results of both Egger's test ( $\mathrm{t}=$ $-0.21, p=0.834)$ and Begg's test $(\mathrm{z}=1.94, p=0.053)$.

\section{Subgroup analyses}

We further examined pooled prevalence according to the subtypes of CLO, CLP, and CPO. For the 92 studies that included 7511 perinatal infants with CLO, the pooled prevalence was $0.56 \%$ o $(95 \% \mathrm{CI}, 0.50-0.63)$. Another 84 studies that included 8463 perinatal infants with CLP gave a pooled prevalence of $0.82 \%$ o $(95 \% \mathrm{CI}, 0.73-0.90)$, and a pooled prevalence of $0.27 \%$ ( $95 \%$ CI, $0.24-0.30)$ were calculated from 42 studies, including 1959 perinatal infants with CPO.

To further characterize the potential impact to the pooled prevalence from the different sample sizes of the included studies, meta-analysis was stratified by sample size. Pooled prevalence estimates were: 0.99\% (95\% CI, 0.57-1.43) for sample sizes more than 1000000 individuals; 1.55\% (95\% CI, 1.34-1.76) for sample sizes between 100000 and $1000000 ; 1.67 \%$ (95\% CI, 1.56-1.78) for sample sizes between 10000 and 100000; and 2.17\% (95\% CI, 1.62-2.72) for sample sizes less than 10000 (Figure 2).

According to hospital level, the results were classified as follows: primary hospital $(1.72 \%, 95 \% \mathrm{CI}$, 1.46-1.97), secondary hospital (1.57\%o, 95\% CI, 1.42 $1.72)$, and tertiary hospital (1.72\%o, 95\% CI, 1.47-1.97). Prevalence of NSOFC by year groups of before 2000, 2000-2009, and 2010 to date were $1.78 \%$ (95\% CI, 1.66 $-1.91), 1.66 \%$ o (95\% CI, 1.44-1.89), and $1.40 \%$ o $(95 \%$ CI, 1.17-1.63), respectively (Figure 2). Although there were no significant time trends, the trend line showed that there was a progressively decreasing of prevalence over the year. The decreasing was also reflected in each hospital level, including primary-, secondary- and tertiary-hospital (Figure 3). In subgroup analysis based on socioeconomic status, the prevalence was highest in low level socioeconomic condition $(1.95 \%$, 95\% CI, 1.82-2.09), followed by middle level (1.65\%, 95\% CI, $1.52-1.78)$, and high level (1.46\%o, 95\% CI, 1.24-1.68).

Regarding different study location, fifty-nine and forty-seven studies offered the prevalence of NSOFC in inland and coastal area, respectively. The prevalence was $1.79 \%$ o (95\% CI, 1.67-1.90) in inland area perinatal infants, and coastal area perinatal infants seemed to have a lower prevalence with an estimation of $1.49 \%$
(95\% CI, 1.27-1.71). Among study regions, the highest prevalence was $2.19 \%$ o $(95 \% \mathrm{CI}, 1.99-2.40)$ in northwest China, followed by southwest China $(1.97 \%$ o (95\% CI, 1.69-2.56)), then north China (1.72\%o (95\% CI, 1.46$1.98)$ ), south China (1.65\% (95\% CI, 1.21-2.09)), central China (1.52\% (95\% CI, 1.33-1.72)), and northeast China (1.47\% (95\% CI, 1.20-1.74)), and east China had the lowest prevalence (1.39\%o (95\% CI, 1.23-1.56)). Further subgroup analysis found that the highest pooled prevalence of NSOFC among perinatal infants was 4.70\% in Hainan, and the lowest was $0.98 \%$ in Shandong. There were significant varied with the provinces (Figure 4).

Sample size, survey year, hospital level, and study region were entered into the meta-regression, which yielded a significant model $(\mathrm{F}=4.11, p=0.0040)$ that explained $13.59 \%$ of the variance. Meanwhile, we found that survey year and study region were significantly associated with heterogeneity in this meta-analysis. The t-values were 2.27 and -2.58; and the $p$-values were 0.025 and 0.011 for the two variables, respectively.

\section{DISCUSSION}

To the best of our knowledge, this is the first systematic review and meta-analysis based on Chinese hospital-based congenital anomalies surveillance systems to determine the national prevalence and its characteristics of non-syndromic orofacial clefts (NSOFC). The present systematic review included 110 articles, involving 15,094,978 Chinese perinatal infants in all 31 provinces, to demonstrate the prevalence of NSOFC and its subgroups, cleft lip only (CLO), cleft lip and palate (CLP), and cleft palate only (CPO). The prevalence of NSOFC was $1.67 \%$ o (95\% CI, 1.53-1.82). Further results showed that prevalence estimates for CLP was highest (0.82\%o), followed by CLO (0.56\%) and CPO (0.27\%o). Given the approximate 2016 population estimates of 17 million perinatal infants [16], this rate amounts to 28390 suffers of NSOFC annually in China.

NSOFC is one of the most common congenital birth defects in perinatal infants and imposes a substantial physical and financial burden on affected individuals and families $[3,6]$. Fetuses with NSOFC usually could be identified by ultrasound. Prenatal transabdominal sonographic could detect the defects during the second trimester (14-28 weeks) of pregnancy [17]. The detection rate can reach $100 \%$ [18], however, the accuracy is highly variable and dependent on the experience of the sonographer, gestational age, the types and fetal position [19]. The sex ratio (male : female) was reported about 2:1 in white population [1] and about 1.25:1 in Chinese population [20]. About seven in ten $(68 \%)$ NSOFC infants were suffered from malnutrition, which is also a major cause of the high mortality of infants [21].

NSOFC is generally classified as CLP, CLO, or CPO. Because CPO is less noticeable externally, the 
prevalence of $\mathrm{CPO}$ might underestimate in perinatal infants in initial survey studies. Findings from this study showed that the prevalence of CPO accounted for only a half of CLO and one third of CLP in Chinese perinatal infants. Meanwhile, previous studies $[6,8]$ were also found CLP high compared to CLO, which were similar to our result. A possible explanation for this difference is that cleft hard palate is often accompanied by a cleft lip [1]. Furthermore, difficulties in identifying perinatal infants with not obvious cleft lip are likely to lead to underascertainment at the beginning of the studies [22].

We found that the prevalence of NSOFC reduced gradually from 2.17 to 0.99 per 1000 perinatal infants with the increasing of sample size, which plays an important role in epidemiology surveys. Small sample sizes are more likely to lead to instable results, especially in the calculation of prevalence in rare diseases [23]. Systematic review and meta-analysis provides a scientific and logical method to

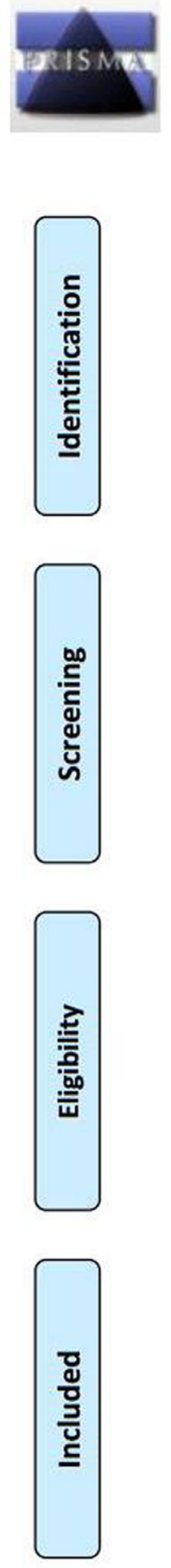

\section{PRISMA 2009 Flow Diagram}

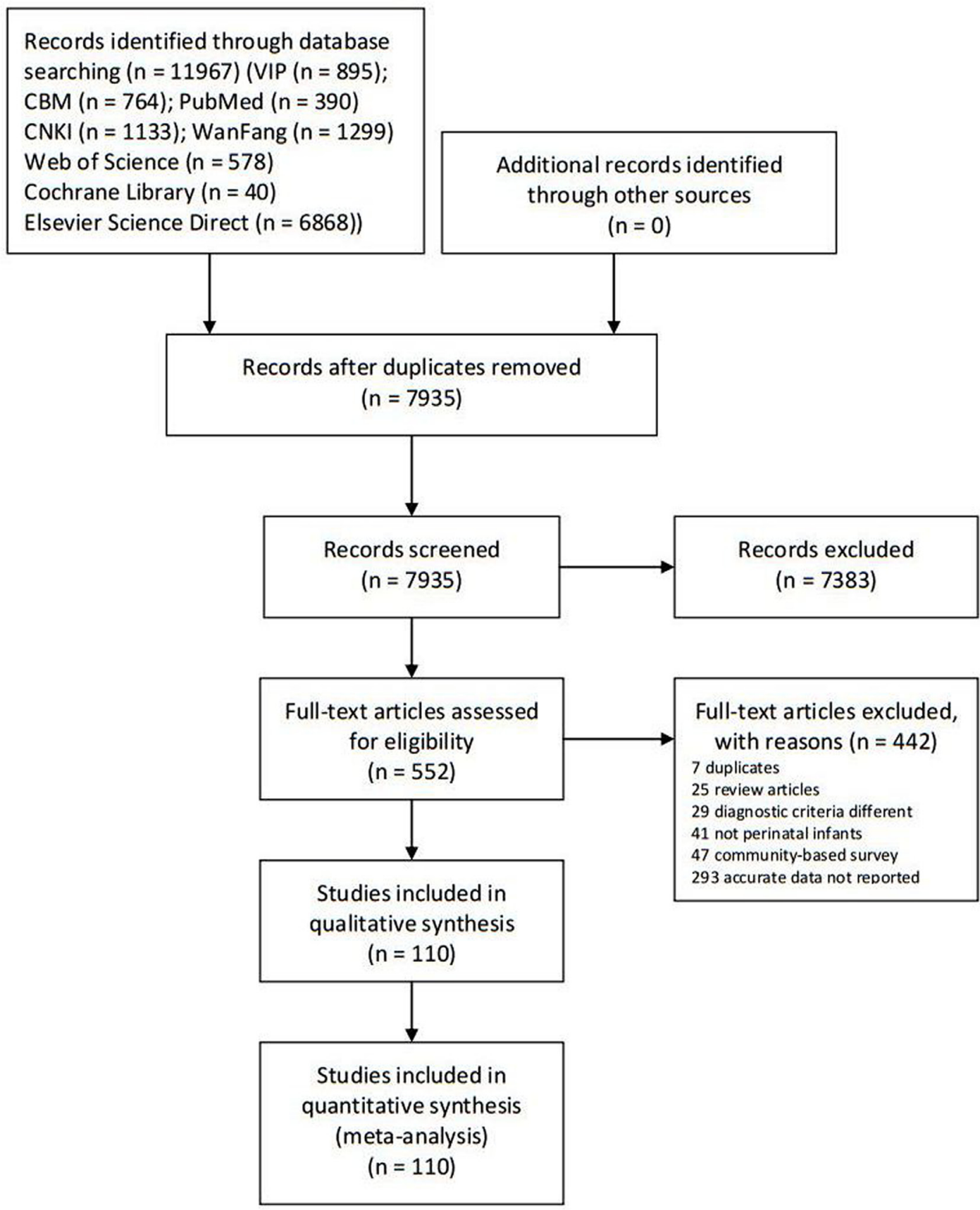

Figure 1: Flowchart of study selection. 
synthesize epidemiological data, and it could enhance the statistical power as well as draw a more reliable conclusion when compared with single studies [24].

A recent systematic review showed that the overall prevalence of orofacial clefts was $1.40 \%$ in Chinese live births [25]. Because few included studies reported relevant data, the study was not estimated the prevalence of cleft types (CLO, CLP, and CPO). And more importantly, Mossey $\mathrm{P}$ has reported that stillbirths have higher prevalence of orofacial clefts than live births [26]. Thus, the prevalence rate of orofacial clefts in live births is generally lower than in perinatal infants. Our results further confirmed Mossey P's findings. Because of the difference in participants, the results, including region, socioeconomic status, sample size, will be different between the two articles.
Genetic and epidemiological studies have clarified that the etiology of NSOFC is involving genetic and environmental risk factors [27-30]. Findings of genomewide and genome-wide meta-analyses have suggested various loci could have a causal role in NSOFC, including 1p22.1: rs560426, 1q32.2: rs861020, 8q24: rs987525, 10q25: rs7078160, and 13q31.1: rs8001641 [28, 29]. About 30\% NSOFC cases can be contributed to these various locus [1, 28, 29]. In addition, epidemiological and experimental data suggested that poor nutrition, maternal tobacco smoking, maternal alcohol use, viral infection, and glucocorticoid exposure were risk factors for NSOFC [31-33]. Maternal smoking during pregnancy has been associated with increased risk of NSOFC, with a population-attributable risk as high as $20 \%$ [34, 35].

\begin{tabular}{|c|c|c|c|c|c|c|}
\hline Outcome & $\begin{array}{c}\text { No of } \\
\text { Studies }\end{array}$ & $\begin{array}{l}\text { No of } \\
\text { NSOFC }\end{array}$ & $\begin{array}{c}\text { No of } \\
\text { Perinatal Infants } \\
\end{array}$ & $\begin{array}{c}\text { Prevalence }(95 \% \mathrm{CI}) \\
(\text { per } 1000)\end{array}$ & $I^{2}(\%)$ & $\begin{array}{c}\text { Prevalence }(95 \% \text { CI }) \\
(\text { per } 1000)\end{array}$ \\
\hline NSOFC & 106 & 14164 & 9476601 & $r+1$ & 97.3 & $1.67(1.53-1.82)$ \\
\hline \multicolumn{7}{|l|}{ Subtypes } \\
\hline $\mathrm{CLO}$ & 92 & 7511 & 13354899 & Het & 96.6 & $0.56(0.50-0.63)$ \\
\hline CLP & 84 & 8463 & 11369412 & tr-1 & 97.0 & $0.82(0.73-0.90)$ \\
\hline $\mathrm{CPO}$ & 61 & 2272 & 9818549 & H & 89.2 & $0.27(0.24-0.30)$ \\
\hline \multicolumn{7}{|l|}{ Sample-Size } \\
\hline Less than 10000 & 5 & 61 & 27452 & $\longrightarrow$ & 0 & $2.17(1.62-2.72)$ \\
\hline $10000-100000$ & 84 & 5336 & 3241308 & 1 & 84.7 & $1.67(1.56-1.78)$ \\
\hline $100000-1000000$ & 15 & 6128 & 3802906 & $\mapsto$ & 96.5 & $1.55(1.34-1.76)$ \\
\hline More than 1000000 & 2 & 2639 & 2404935 & $\longmapsto$ & 99.9 & $0.99(0.57-1.43)$ \\
\hline \multicolumn{7}{|l|}{ Hospital-Level } \\
\hline Primary & 17 & 1128 & 676895 & $\longmapsto$ & 81.6 & $1.72(1.46-1.97)$ \\
\hline Secondary & 44 & 6404 & 4004302 & $\mapsto \dot{4}$ & 92.0 & $1.57(1.42-1.72)$ \\
\hline Tertiary & 45 & 6632 & 4795404 & $\longmapsto$ & 98.2 & $1.72(1.47-1.97)$ \\
\hline \multicolumn{7}{|l|}{ Survey-Year } \\
\hline Before 2000 & 36 & 2771 & 1554961 & - & 70.5 & $1.78(1.66-1.91)$ \\
\hline $2000-2009$ & 53 & 8612 & 6007146 & $\mapsto$ & 98.2 & $1.66(1.44-1.89)$ \\
\hline After 2010 & 17 & 2781 & 1914494 & $\longmapsto$ & 94.1 & $1.40(1.17-1.63)$ \\
\hline \multicolumn{7}{|l|}{ Socioeconomic-Status } \\
\hline High level & 45 & 7243 & 5475990 & $\mapsto-1$ & 98.2 & $1.46(1.24-1.68)$ \\
\hline Middle level & 31 & 4063 & 2530873 & $\stackrel{1}{H}$ & 82.2 & $1.65(1.52-1.78)$ \\
\hline Low level & 30 & 2858 & 1469738 & $\mapsto$ & 60.6 & $1.95(1.82-2.09)$ \\
\hline \multicolumn{7}{|l|}{ Study-Location } \\
\hline Inland & 59 & 6732 & 3914536 & $i$ & 83.6 & $1.79(1.67-1.90)$ \\
\hline Coastal & 47 & 7432 & 5562065 & $\mapsto$ & 98.2 & $1.49(1.27-1.71)$ \\
\hline \multicolumn{7}{|l|}{ Study-Region } \\
\hline East & 29 & 2413 & 1800294 & $\mapsto-1$ & 89.0 & $1.39(1.23-1.56)$ \\
\hline South & 16 & 5546 & 4091647 & $\longmapsto$ & 99.3 & $1.65(1.21-2.09)$ \\
\hline North & 12 & 1136 & 660202 & $\longmapsto$ & 81.9 & $1.72(1.46-1.98)$ \\
\hline Central & 13 & 1163 & 770923 & $\mapsto-4$ & 74.4 & $1.52(1.33-1.72)$ \\
\hline Northeast & 10 & 1298 & 871189 & $\longmapsto$ & 90.5 & $1.47(1.20-1.74)$ \\
\hline Southwest & 10 & 763 & 390728 & $\longmapsto$ & 71.4 & $1.97(1.69-2.56)$ \\
\hline \multirow[t]{2}{*}{ Northwest } & 16 & 1845 & 891618 & $\mapsto$ & 58.7 & $2.19(1.99-2.40)$ \\
\hline & & & 0.0 & $\begin{array}{lllll}0.50 & 1.00 & 1.50 & 2.00 & 2.50\end{array}$ & 3.00 & \\
\hline
\end{tabular}

Figure 2: Results of subgroup analysis for the pooled prevalence of NSOFC in Chinese perinatal infants. 
Stratified by time spans, the highest prevalence of NSOFC was observed before 2000, and the lowest in 2010 to date. Although the results were not significantly different, it seems that there is a decreasing trend of prevalence with time. After further study, the trend line also showed there was a progressively decreasing of prevalence over the year. On one hand, the high prevalence appeared before 2009 might be partly explained by complete ascertainment through the registry and improved prenatal detection [36]. On the other hand, the decreasing trend after 2010 might be attributed that the government refocused the importance of the primary prevention for women at childbearing age [37]. Most cities [38-40] have switched from prenatal care to preconceptional or periconceptional care through the Local Family Planning System and Maternal and Child Health Care System in recent years. In addition, prenatal diagnostic techniques, including fetal ultrasound, fetal echocardiography, and karyotyping following amniocentesis and chorionic villus sampling have been available to diagnose severe structural defects before early second trimester (usually before 22 gestational weeks) [41], and virtually all mothers who carry a fetus affected by a severe malformation would choose elective termination [42].

China is a vast, multiracial country, and in this study we found significant differences in the prevalence of NSOFC in perinatal infants by territories (inland vs coastal, and between regions and provinces). Inland perinatal infants have a higher prevalence compared with that of coastal area, and prevalence varied substantially among the 7 regions and 31 provinces. The prevalence of provinces spans from 0.98 to 4.70 per 1000 perinatal infants. The highest reported rates were in Hainan, Chongqing, and Qinghai, with prevalence rates of 4.70, 3.17 , and 3.00 per 1000 perinatal infants, respectively. The lowest reported rates were in Shandong, Fujian, and Liaoning, with prevalence of $0.98,1.06$, and 1.29 per 1000 perinatal infants, respectively. It is likely that many factors could contribute to this geographic difference, such as environmental pollution, economic status, health service status, and diagnostic level [11].

Most of the minorities are concentrated in northwest and southwest developing provinces in China [43]. Compared to east developed provinces, there are some differences in their genetics and lifestyle [44, 45]. Meanwhile, prenatal diagnostic techniques vary greatly among provinces and hospitals in China. The developed provinces have made the greatest efforts in prenatal screening and diagnosis and mothers would choose elective termination before 22 gestational weeks when they found a fetus affected by a severe malformation [42]. Meanwhile, most coastal cities attached importance to the primary prevention in childbearing age women, which could reduce the occurrence of birth defects [39]. The above reasons can be partially explained why there is the different prevalence based on different regions. To reduce the incidence of NSOFC, preconception care and antenatal screening should be promoted in every women of reproductive age.

There are many strengths in our systematic review and meta-analysis. No systematic review and metaanalysis has been performed to synthesize the prevalence of NSOFC in Chinese perinatal infants. Our study is the first to adhere to PRISMA guidelines to quantify

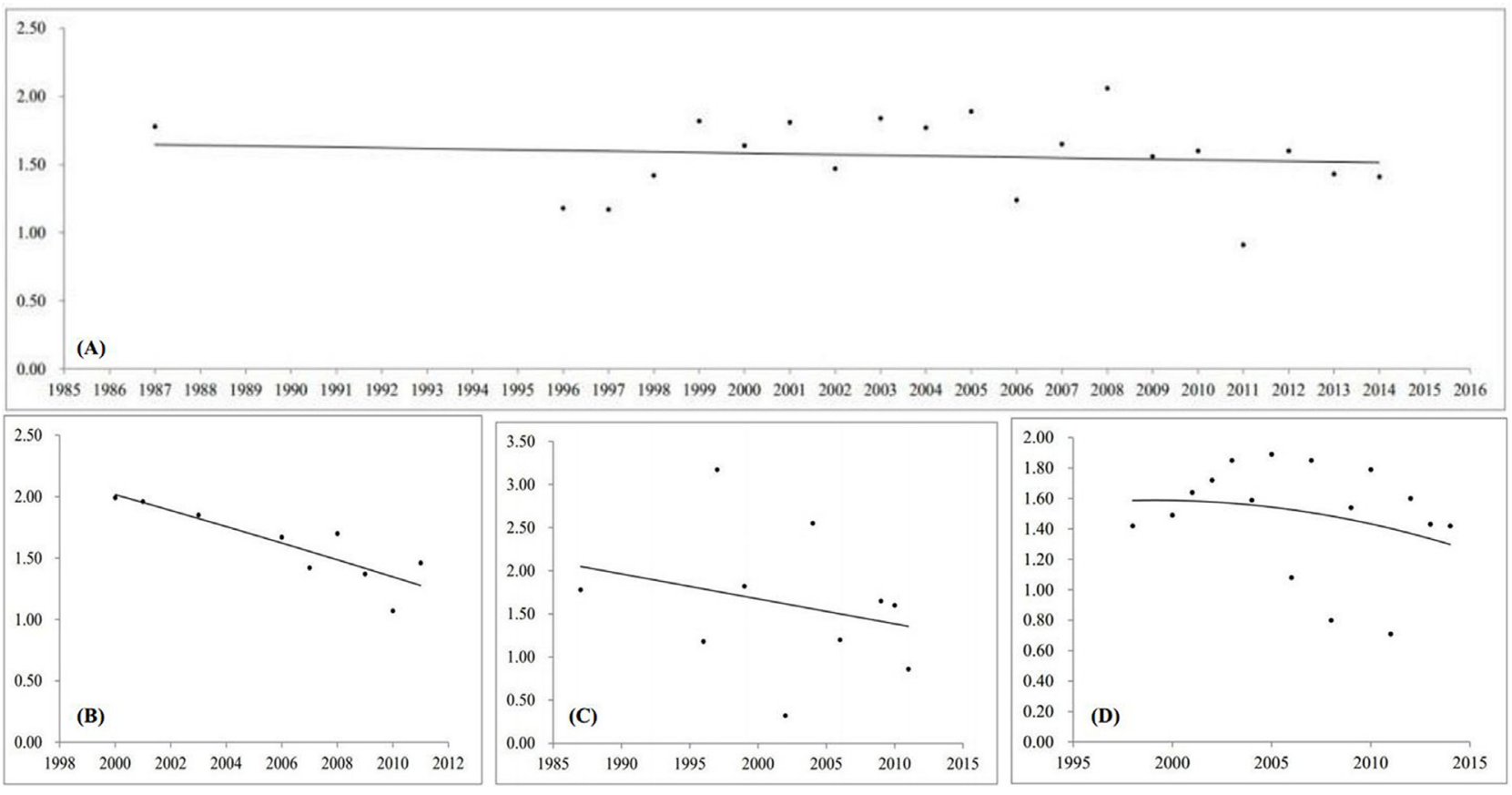

Figure 3: Trend lines of the prevalence of NSOFC over the year ( $x$ axes is the survey year, $y$ axes is prevalence (per 1000)). (A) all of Chinese perinatal infants, (B) by primary hospital, (C) by secondary hospital, (D) by tertiary hospital. 
prevalence estimates derived from a comprehensive search strategy. It has a relatively large number of perinatal infants which including 15,094,978 across all 31 provinces in mainland China, and this allows us to get a stable result. Meanwhile, overall quality of the included studies was acceptable, publication bias tests did not show potential risk, and sensitivity analysis was not substantially altered.

Despite the strengths of this study, several limitations need to be considered in interpreting with future research. On one hand, like other similar prevalence meta-analyses [46-49], heterogeneity usually existed, and was not fully resolved by subgroup and regression model, although survey year and study region could explain a part of its heterogeneity. On the other hand, it should be noted that due to mild disorders, especially in $\mathrm{CPO}$, undiagnosed in utero or lack of external visibility at birth may be underestimate the true prevalence. Meanwhile, the sources of data were all captured from hospital-based surveillance systems, which could also underestimate the prevalence compared with population-based survey. In addition, because of the limited information in included studies, this prevalence meta-analysis could not provide more detailed results, such as age of infants when disease was diagnosed, sex ratio of infants diagnosed with NSOFC, and trend lines of prevalence in each province.

In this systematic review, the pooled estimate of the prevalence of NSOFC was 1.67 per 1000 in Chinese perinatal infants, varying with provinces. The result will serve as a baseline for future assessment of the overall effectiveness of the NSOFC control in China, and will also could support and inform health policy for planning and helping health debates.

\section{MATERIALS AND METHODS}

\section{Protocol and registration}

Similar as our previous studies [50-52], this metaanalysis was also strictly performed according to the guidelines in the Preferred Reporting Items for Systematic Reviews and Meta-Analyses (PRISMA) statement (Supplementary Table 2) [53]. The protocol for this systematic review was pre-registered in the PROSPERO International Prospective Register of systematic reviews (http://www.crd.york.ac.uk/PROSPERO/), and the registration number is CRD42017062293.

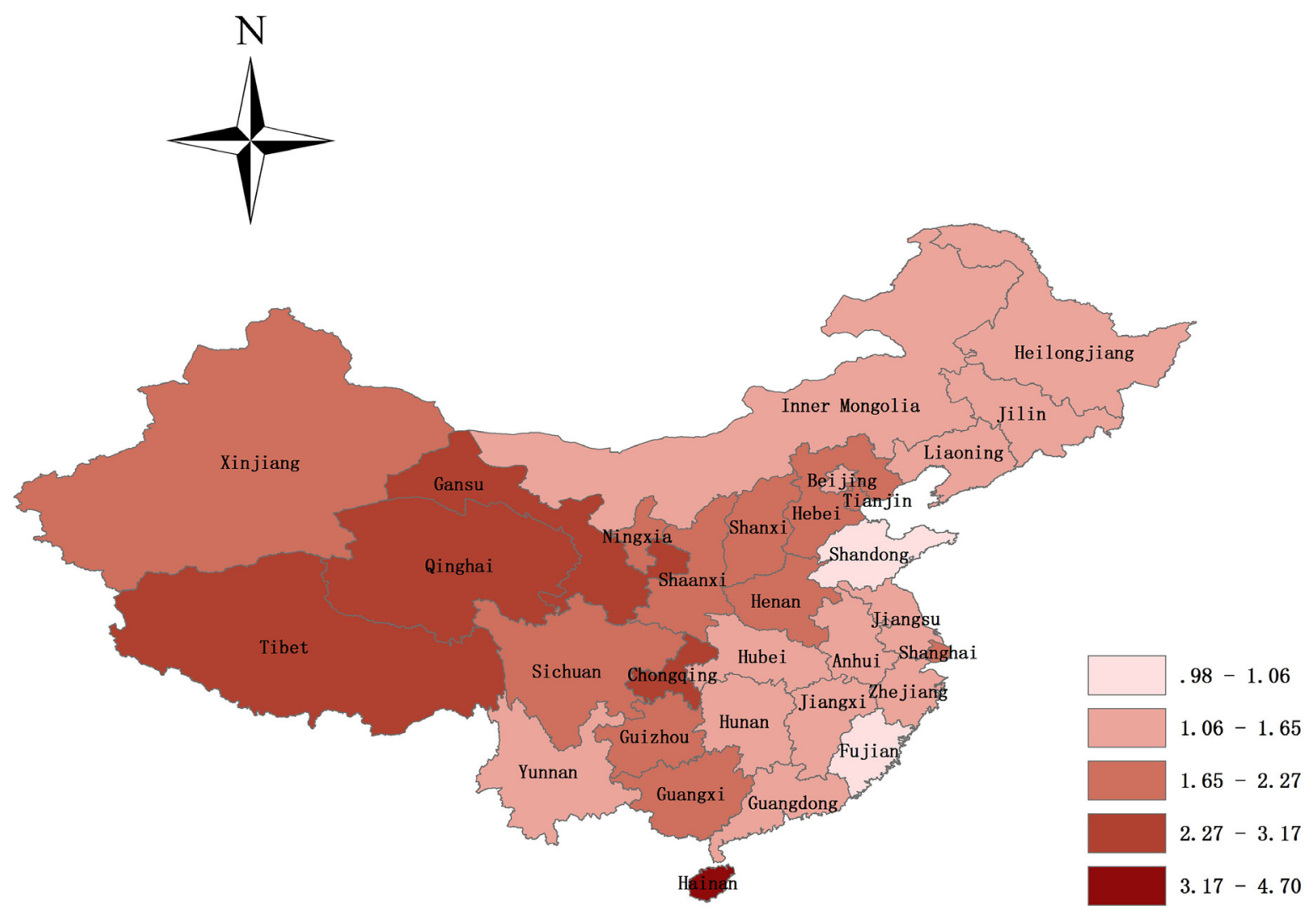

Figure 4: Map of the prevalence of NSOFC in Mainland Chinese perinatal infants (not including Hong Kong, Macao, and Taiwan). The pooled prevalence of NSOFC among perinatal infants was $4.70 \%$ in Hainan, followed by $3.17 \%$ in Chongqing, $3.00 \% 0$ in Qinghai, 2.84\%o in Tibet, 2.46\% in Gansu, 2.27\% in Guizhou, 2.18\%o in Ningxia, 2.11\%o in Shaanxi, 1.97\%o in Shanxi and Sichuan, 1.94\%o in Henan, 1.80\%o in Xinjiang, 1.78\%o in Hebei, 1.77\%o in Shanghai, 1.74\%o in Tianjin, 1.72\%o in Guangxi, 1.65\%o in Anhui, 1.62\%o in Jilin, $1.57 \%$ in Zhejiang, 1.55\% in Jiangxi, 1.54\%o in Inner Mongolia, 1.53\%o in Yunnan, 1.51\%o in Heilongjiang, 1.45\%o in Hunan, $1.44 \%$ in Guangdong, 1.44\%o in Hubei, 1.37\% in Jiangsu, 1.35\% in Beijing, 1.29\%o in Liaoning, 1.06\%o in Fujian, and the lowest is $0.98 \%$ o in Shandong. 


\section{Search strategy}

Data for this meta-analysis was comprehensively identified by searching four English databases (PubMed, Elsevier Science Direct, Web of Science, Cochrane Library) and four Chinese databases (Chinese Biological Medical Literature database (CBM), Chinese National Knowledge Infrastructure (CNKI), Chongqing VIP (VIP), WanFang) from inception to April 2017. Search strategy was listed as follows: ("birth defect" OR "orofacial clefts" OR "cleft lip" OR "cleft lip and palate" OR "cleft palate") AND ("prevalence" OR "incidence" OR "epidemiology" OR "Survey") AND ("China" OR "Chinese"). In addition, snowball searching of reference lists was also conducted to find further relevant articles.

Language was restricted to either Chinese or English. Two researchers (L Liu and G Tian) independently reviewed all titles and abstracts. The complete relevant articles were downloaded for further screening. If the same data was reported in more than one publication, only the paper with a better quality was included. Any uncertainty was settled by discussion with the third participant (D Fan). In addition, we performed a further search by perusing the references of review articles and e-mailing the authors for full-text articles to include all available data in this meta-analysis.

\section{Inclusion and exclusion criteria}

Articles fulfilling the following criteria were considered eligible for inclusion: 1) cross-sectional studies examining prevalence of NSOFC (including CLO, CLP, and/or CPO) in perinatal infants (between 28 weeks of gestation and 7 days after birth) in mainland China (not including Hong Kong, Taiwan, and Macao); 2) the diagnosis was based on the Chinese national criteria of birth defects [9]; 3) sample sizes from hospital-based survey; 4) publications in full text either written in English or in Chinese. Studies conducted in specific populations (e.g., children, adolescents, and the elderly) or special settings (e.g. community-based, population-based) and studies used census sampling were excluded.

\section{Data extraction}

The available data extraction was independently performed by two researchers using a standardized form, and the disagreement was resolved through discussion. The key data extraction sheet included: first author, year of publication, year of the study conducted, province where the survey was conducted, number of perinatal infants, number of NSOFC (CLO, CLP, and/or CPO).

\section{Quality assessment}

In line with other and our previously studies [54, 55], two researchers (SZ Wu and W Wang) independently assessed the risk of bias and methodological quality of each study using the Newcastle-Ottawa Score (NOS) for evaluating the quality of observational epidemiological studies [56].

\section{Statistical analysis}

In this meta-analysis, all statistical analyses were undertaken with STATA software, Version 12.0 (Stata Corporation, College Station, Texas, USA). Cochran's Q statistic and $I^{2}$ index were calculated to assess the heterogeneity of the prevalence across studies. A p < 0.05 or the $I^{2}$ statistic $>50 \%$ indicated heterogeneity in the effect size [57]. Due to heterogeneity across studies, a random effect model was used for this meta-analysis [58]. To determine the sources of heterogeneity, subgroup analyses and meta-regression were conducted based on different categories: sample size $(<10000,10000$ - 100000, $100000-1000000$, and > 1000000), year of data collection (before 2000, 2000-2009, and after 2010), hospital level (primary, secondary, and tertiary), socioeconomic status [59], study location (inland and coastal), and study region (east, north, northwest, northeast, southwest, central, and south). Moreover, in order to more clearly display area distribution, the prevalence of each province was also calculated. Maps were drawn using ArcMap version 10.2 (Environmental Systems Research Institute, Redlands, CA). To test the robustness of the result on the overall prevalence estimates, sensitivity analysis was performed by serially excluding each included studies. Publication bias was estimated by testing for funnel plot asymmetry and using Egger's and Begg's test. All $p$-values were two-sided, and $p<0.05$ was regarded statistically significant.

\section{ACKNOWLEDGMENTS}

We appreciate the efforts of all the researchers whose articles were included in this study.

\section{CONFLICTS OF INTEREST}

The authors declare no conflicts of interest.

\section{GRANT SUPPORT}

The authors did not receive any financial support.

\section{REFERENCES}

1. Mossey PA, Little J, Munger RG, Dixon MJ, Shaw WC. Cleft lip and palate. Lancet. 2009; 374:1773-1785.

2. Allori AC, Mulliken JB, Meara JG, Shusterman S, Marcus JR. Classification of Cleft Lip/Palate: Then and Now. Cleft Palate Craniofac J. 2017; 54:175-188. 
3. Burg ML, Chai Y, Yao CA, Magee W 3rd, Figueiredo JC. Epidemiology, Etiology, and Treatment of Isolated Cleft Palate. Front Physiol. 2016; 7:67.

4. Stothard KJ, Tennant PW, Bell R, Rankin J. Maternal overweight and obesity and the risk of congenital anomalies: a systematic review and meta-analysis. Jama. 2009; 301:636-650.

5. Zucchero TM, Cooper ME, Maher BS, Daack-Hirsch S, Nepomuceno B, Ribeiro L, Caprau D, Christensen K, Suzuki Y, Machida J, Natsume N, Yoshiura K, Vieira AR, et al. Interferon regulatory factor 6 (IRF6) gene variants and the risk of isolated cleft lip or palate. N Engl J Med. 2004; 351:769-780.

6. Watkins SE, Meyer RE, Strauss RP, Aylsworth AS. Classification, epidemiology, and genetics of orofacial clefts. Clin Plast Surg. 2014; 41:149-163.

7. Carlson L, Hatcher KW, Vander Burg R. Elevated infant mortality rates among oral cleft and isolated oral cleft cases: a meta-analysis of studies from 1943 to 2010. Cleft Palate Craniofac J. 2013; 50:2-12.

8. Kadir A, Mossey PA, Blencowe H, Moorthie S, Lawn JE, Mastroiacovo P, Modell B. Systematic Review and Metaanalysis of the Birth Prevalence of Orofacial Clefts in Lowand Middle-Income Countries. Cleft Palate Craniofac J. 2017; 54:571-581.

9. Dai L, Zhu J, Liang J, Wang YP, Wang H, Mao M. Birth defects surveillance in China. World J Pediatr. 2011; 7:302-310.

10. Xie D, Yang T, Liu Z, Wang H. Epidemiology of Birth Defects Based on a Birth Defect Surveillance System from 2005 to 2014 in Hunan Province, China. PloS one. 2016; 11:e0147280.

11. Liu QG, Sun J, Xiao XW, Song GR. Birth defects data from surveillance hospitals in Dalian city, China, 2006-2010. J Matern Fetal Neonatal Med. 2016; 29:3615-3621.

12. Yu M, Ping Z, Zhang S, He Y, Dong R, Guo X. The survey of birth defects rate based on birth registration system. Chin Med J (Engl). 2015; 128:7-14.

13. Li B, Zhang XZ, Ye N, Mu L, Xia JH, Huang XJ, He SL. Study on the changes of incidence rates on birth defects through hospital based surveillance program in Guangdong province during 1997-2007. Zhonghua liu xing bing xue za zhi. 2008; 29:1101-1105. [Article in Chinese].

14. Zheng ZJ, Gong X. Analysis on perinatal mortality and related factors in Hainan from 2005 to 2010. MCHCC. 2011; 26:3261-3262.

15. Yin YT, Xiu XH, Yuan L. Birth deffects in Qingdao: An analysis of 1085 neonates. Med J Qilu. 2013; 28:530-532.

16. National Health and Family Planning Commission of the People's Republic of China. http://newscctvcom/2016/03/08/ VIDEZCsj9eLplF28ud5pZL7M160308shtml.

17. Stoll C, Dott B, Alembik Y, Roth M. Evaluation of prenatal diagnosis of cleft lip/palate by foetal ultrasonographic examination. Ann Genet. 2000; 43:11-14.

18. Maarse W, Berge SJ, Pistorius L, van Barneveld T, Kon M, Breugem C, Mink van der Molen AB. Diagnostic accuracy of transabdominal ultrasound in detecting prenatal cleft lip and palate: a systematic review. Ultrasound Obstet Gynecol. 2010; 35:495-502.

19. Kim DW, Chung SW, Jung HD, Jung YS. Prenatal ultrasonographic diagnosis of cleft lip with or without cleft palate; pitfalls and considerations. Maxillofac Plast Reconstr Surg. 2015; 37:24.

20. Lili Y, Jian M, Junpeng G, Kun Z, Jinfang Z, Yongqing H. Association between non-syndromic cleft lip with or without cleft palate and environmental factors in Ningxia. Hua xi kou qiang yi xue za zhi. 2017; 35:291-295. [Article in Chinese].

21. Tungotyo M, Atwine D, Nanjebe D, Hodges A, Situma M. The prevalence and factors associated with malnutrition among infants with cleft palate and/or lip at a hospital in Uganda: a cross-sectional study. BMC Pediatr. 2017; 17:17.

22. Butali A, Adeyemo WL, Mossey PA, Olasoji HO, Onah II, Adebola A, Efunkoya, Akintububo A, James O, Adeosun OO, Ogunlewe MO, Ladeinde AL, Mofikoya BO, et al. Prevalence of orofacial clefts in Nigeria. Cleft Palate Craniofac J. 2014; 51:320-325.

23. Giraudeau B, Higgins JP, Tavernier E, Trinquart L. Sample size calculation for meta-epidemiological studies. Stat Med. 2016; 35:239-250.

24. Stroup DF, Berlin JA, Morton SC, Olkin I, Williamson GD, Rennie D, Moher D, Becker BJ, Sipe TA, Thacker SB. Metaanalysis of observational studies in epidemiology: a proposal for reporting. Meta-analysis Of Observational Studies in Epidemiology (MOOSE) group. Jama. 2000; 283:2008-2012.

25. Wang M, Yuan Y, Wang Z, Liu D, Wang Z, Sun F, Wang P, Zhu H, Li J, Wu T, Beaty TH. Prevalence of Orofacial Clefts among Live Births in China: A Systematic Review and MetaAnalysis. Birth Defects Res. 2017; 109:1011-1019.

26. Mossey P. Epidemiology underpinning research in the aetiology of orofacial clefts. Orthod Craniofac Res. 2007; 10:114-120.

27. Wang F, Jiang Y, Yang S, Liu Q, Lin J, Zhang H. Associations between the NOGGIN rs227731 polymorphism and NSCL/P risk may be associated with ethnicities: A meta-analysis. Birth Defects Res. 2017; 109:445-451.

28. Sun Y, Huang Y, Yin A, Pan Y, Wang Y, Wang C, Du Y, Wang M, Lan F, Hu Z, Wang G, Jiang M, Ma J, et al. Genome-wide association study identifies a new susceptibility locus for cleft lip with or without a cleft palate. Nat Commun. 2015; 6:6414.

29. Ludwig KU, Mangold E, Herms S, Nowak S, Reutter H, Paul A, Becker J, Herberz R, AlChawa T, Nasser E, Bohmer AC, Mattheisen M, Alblas MA, et al. Genome-wide meta-analyses of nonsyndromic cleft lip with or without cleft palate identify six new risk loci. Nat Genet. 2012; 44:968-971.

30. Chung MK, Lao TT, Ting YH, Leung TY, Lau TK, Wong TW. Environmental factors in the first trimester and risk of oralfacial clefts in the offspring. Reprod Sci. 2013; 20:797-803.

31. Sabbagh HJ, Hassan MH, Innes NP, Elkodary HM, Little J, Mossey PA. Passive smoking in the etiology of nonsyndromic orofacial clefts: a systematic review and metaanalysis. PloS one. 2015; 10:e0116963. 
32. Pei L, Kang Y, Cheng Y, Yan H. The Association of Maternal Lifestyle with Birth Defects in Shaanxi Province, Northwest China. PloS one. 2015; 10:e0139452.

33. Bille C, Olsen J, Vach W, Knudsen VK, Olsen SF, Rasmussen K, Murray JC, Andersen AM, Christensen K. Oral clefts and life style factors--a case-cohort study based on prospective Danish data. Eur J Epidemiol. 2007; 22:173-181.

34. Honein MA, Rasmussen SA, Reefhuis J, Romitti PA, Lammer EJ, Sun L, Correa A. Maternal smoking and environmental tobacco smoke exposure and the risk of orofacial clefts. Epidemiology. 2007; 18:226-233.

35. Little J, Cardy A, Munger RG. Tobacco smoking and oral clefts: a meta-analysis. Bull World Health Organ. 2004; 82:213-218.

36. Chiu RW, Lo YM. Noninvasive prenatal diagnosis empowered by high-throughput sequencing. Prenat Diagn. 2012; 32:401-406.

37. Liu J, Gao L, Zhang Y, Jin L, Li Z, Zhang L, Meng Q, Ye R, Wang L, Ren A. Plasma folate levels in early to mid pregnancy after a nation-wide folic acid supplementation program in areas with high and low prevalence of neural tube defects in China. Birth Defects Res A Clin Mol Teratol. 2015; 103:501-508.

38. Shang XX, Wang ZP, Zhao ZT. Progress on the role of vitamin $\mathrm{B} 12$ in primary prevention of neural tube defects. Zhonghua liu xing bing xue za zhi. 2010; 31:579-583. [Article in Chinese].

39. Gong R, Wang ZP, Wang M, Gao LJ, Zhao ZT. Effects of folic acid supplementation during different pregnancy periods and relationship with the other primary prevention measures to neural tube defects. J Matern Fetal Neonatal Med. 2016; 29:3894-3901.

40. Yang W, Zeng L, Cheng Y, Chen Z, Wang X, Li X, Yan $\mathrm{H}$. The effects of periconceptional risk factor exposure and micronutrient supplementation on birth defects in Shaanxi Province in Western China. PloS one. 2012; 7:e53429.

41. Wang Y, Cai A, Sun J, Li T, Wang B, Li J. Prenatal diagnosis of penoscrotal transposition with 2- and 3-dimensional ultrasonography. J Ultrasound Med. 2011; 30:1397-1401.

42. Zhu BS, Su J, Lu XH, He J, Zhu S, Jiao CX, Zhang JM, Tang XH, Tao Y, Lin KP, Chen H, Li SY. Study on key techniques and intervention in reducing birth defects. Zhonghua fu chan ke za zhi. 2011; 46:658-663. [Article in Chinese].

43. Chinese Nationalities. http://wwwgoven/ guoqing/2005-07/26/content_2663470htm.

44. Zhao D, Li Y, Zheng L. Ethnic inequalities and sex differences in body mass index among tibet minorities in China: implication for overweight and obesity risks. Am J Hum Biol. 2014; 26:856-858.

45. Song F, Luo HB, Hou YP. Population data of 21 non-CODIS STR loci in the Chinese Uygur ethnic minority. Forensic Sci Int Genet. 2014; 13:e1-2.

46. Fan D, Li S, Wu S, Wang W, Ye S, Xia Q, Liu L, Feng J, Wu S, Guo X, Liu Z. Prevalence of abnormally invasive placenta among deliveries in mainland China: A PRISMAcompliant Systematic Review and Meta-analysis. Medicine (Baltimore). 2017; 96:e6636.
47. Fan D, Xia Q, Wu S, Liu L, Yu Z, Wang W, Wu S, Guo $\mathrm{X}$, Liu Z. Prevalence of prenatally diagnosed congenital cystic adenomatoid malformation among fetuses in China. Oncotarget. 2017; 8:79587-79593. http://doi.org/10.18632/ oncotarget.18579.

48. Yon Y, Mikton CR, Gassoumis ZD, Wilber KH. Elder abuse prevalence in community settings: a systematic review and meta-analysis. Lancet Glob Health. 2017; 5:e147-e156.

49. He C, Liu L, Chu Y, Perin J, Dai L, Li X, Miao L, Kang L, Li Q, Scherpbier R, Guo S, Rudan I, Song P, et al. National and subnational all-cause and cause-specific child mortality in China, 1996-2015: a systematic analysis with implications for the Sustainable Development Goals. Lancet Glob Health. 2017; 5:e186-e197.

50. Fan D, Wu S, Liu L, Xia Q, Wang W, Guo X, Liu Z. Prevalence of antepartum hemorrhage in women with placenta previa: a systematic review and meta-analysis. Sci Rep. 2017; 7:40320.

51. Fan D, Xia Q, Liu L, Wu S, Tian G, Wang W, Wu S, Guo $X$, Liu Z. The Incidence of Postpartum Hemorrhage in Pregnant Women with Placenta Previa: A Systematic Review and Meta-Analysis. PloS one. 2017; 12:e0170194.

52. Fan D, Wu S, Wang W, Xin L, Tian G, Liu L, Feng J, Guo $\mathrm{X}$, Liu Z. Prevalence of placenta previa among deliveries in Mainland China: A PRISMA-compliant systematic review and meta-analysis. Medicine (Baltimore). 2016; 95:e5107.

53. Moher D, Liberati A, Tetzlaff J, Altman DG, Group P. Preferred reporting items for systematic reviews and metaanalyses: the PRISMA statement. Int J Surg. 2010; 8:336-341.

54. Rotenstein LS, Ramos MA, Torre M, Segal JB, Peluso MJ, Guille C, Sen S, Mata DA. Prevalence of Depression, Depressive Symptoms, and Suicidal Ideation Among Medical Students: A Systematic Review and Meta-Analysis. Jama. 2016; 316:2214-2236.

55. Liu L, Fan D, Ding N, Hu Y, Cai G, Wang L, Xin L, Xia Q, Li X, Xu S, Xu J, Yang X, Zou Y, Pan F. The relationship between DRD2 gene polymorphisms (C957T, C939T) and schizophrenia: a meta-analysis. Neurosci Lett. 2014; 583:43-48.

56. Wells GA, Shea B, O'Connell D, Peterson J, Welch V, Losos M, Tugwell P. The Newcastle-Ottawa Scale (NOS) for assessing the quality of nonrandomised studies in metaanalyses. Ottawa Health Research Institute. 2011; http:// www.ohri.ca/programs/clinical_epidemiology/oxford.asp.

57. Fan D, Liu S, Yang T, Wu S, Wang S, Li G, Zeng Z, Duan Z, Xia G, Ye D, Zou Y, Xu S, Xu J, et al. Association between KIR polymorphisms and ankylosing spondylitis in populations: a meta-analysis. Mod Rheumatol. 2014; 24:985-991.

58. DerSimonian R, Laird N. Meta-analysis in clinical trials revisited. Contemp Clin Trials. 2015; 45:139-145.

59. Sun J, Kong W. Research on Comprehensive Evaluation Index of China's Economic Efficiency-Comparative Analysis Based on Panel Data of Eastern, Central and Western Regions. Journal of Beijing Technology and Business University(Social Sciences). 2017; 32:110-119. 